\title{
Smart RISUG: A potential new contraceptive and its magnetic field-mediated sperm interaction
}

\author{
Rakhi K Jha',2 \\ Pradeep K Jha ${ }^{1,3}$ \\ Sujoy K Guha' \\ 'School of Medical Science \\ and Technology, Indian Institute of \\ Technology, Kharagpur, West Bengal, \\ India; ${ }^{2}$ Toxicology Laboratory, \\ Department of Zoology, ChCS \\ University, Meerut, UP, India; \\ ${ }^{3}$ Department of Management studies, \\ VIET, UP Tech. Univ., Lucknow, \\ UP, India
}

\begin{abstract}
The rationale and technique underlying a novel concept of noninvasive fertility control by a new Cuproferrogel contraceptive drug, iron oxide-copper-styrene maleic anhydride-dimethyl sulphoxide $\left(\mathrm{Fe}_{3} \mathrm{O}_{4}-\mathrm{Cu}-\mathrm{SMA}-\mathrm{DMSO}\right)$ composite named 'Smart RISUG' (smart reversible inhibition of sperm under guidance) in presence of pulsed magnetic field (PMF; $1 \mathrm{mT}$ to $800 \mathrm{mT}$ ) is explained. It was synthesized by dispersing iron oxide particles and copper particles into SMA-DMSO (male contraceptive RISUG) and characterized for particle distribution, particle size measurement and transmittance peaks, etc. Interaction of the RISUG particles as well as Smart RISUG particles with Albino rat sperm cell was studied in presence as well as absence of PMF. To find an explanation to increased reaching of the Smart RISUG drug into sperm under influence of magnetic field, the transport properties were characterized by high resolution transmission electron microscopy and atomic force microscopy. Smart RISUG could be mobilized into sperm cell membrane at the PMF, $760 \mathrm{mT}$ in about 50 seconds. Adoption of novel drug Smart RISUG involving new technique may open the pathway for non surgical control of drug distribution, detection and restoration of the normal fertility after removal of the contraceptive from the male/female reproductive tube in presence of electromagnetic field.
\end{abstract}

Keywords: smart RISUG, pulsed magnetic field, noninvasive fertility control, drug distribution, sperm cell

\section{Introduction}

Long acting and reversible fertility control still remains a challenge for the scientific community. Various contraceptive methods, including the use of hormone medications, intrauterine contraceptive devices, barrier contraception, periods of abstaining from sex, and surgery have been developed over the last few decades to prevent fertilization. Yet contraception failure and user dissatisfaction is not decreasing. ${ }^{1}$ An underlying reason for these limitations is that in time the state, both qualitatively and quantitatively, of the drug or device placed inside the body cannot be assessed noninvasively. The medical personnel are therefore not able to give advice regarding the likely period of contraceptive efficacy. Additionally after implantation, the contraceptive agents cannot be controlled and inactivated in a programmed manner from outside the body to restore fertility on the user's demand.

Reversible inhibition of sperm under guidance (RISUG) is an injectable male contraceptive of polyelectrolytic nature currently undergoing extended Phase III clinical trial. ${ }^{2}$ It consists of a hydrogel preparation of copolymer styrene maleic anhydride (SMA) dissolved in dimethyl sulphoxide (DMSO) possessing antimicrobial properties. ${ }^{3,4}$ Long-term vas occlusion with RISUG (SMA-DMSO) has been proved safe $^{5-9}$ and effective. ${ }^{10-13}$ Reversal by injection of sodium bicarbonate solution as solvent has been demonstrated in the rat. ${ }^{14}$ Noninvasive reversal of SMA after a short period of implantation is also possible, ${ }^{5,6,15,16}$ but the noninvasive reversal method after extended period following the RISUG injection is not yet proven. Therefore although 
RISUG is an advance in male contraceptive technology, its inadequate detection properties in imaging and external to the body control calls for the development of an improved contraceptive.

Smart RISUG, the new implant device described in the present paper, is seen in in vitro and rat in vivo studies to give better spermicidal action than RISUG. ${ }^{17}$ Also comprising of iron oxide-copper-styrene maleic anhydride-dimethyl sulphoxide $\left(\mathrm{Fe}_{3} \mathrm{O}_{4}-\mathrm{Cu}-\mathrm{SMA}-\mathrm{DMSO}\right)$ the implant is detectable by $\mathrm{X}$-ray and magnetic imaging and in vivo distribution can be controlled by the application from outside the body with an external pulsed magnetic field (PMF). ${ }^{18}$ The Smart RISUG implant design targets to overcome the two major limitations of RISUG that pertain to control and detection.

The underlying science behind Smart RISUG links with magnetics and polymer science. One phenomena drawn upon for the in vivo control of Smart RISUG is the change of the elasticity of a ferromagnetic polymeric compound with magnetization. ${ }^{19}$ It is known that both the concentration of magnetite particles and the cross-linking density of the ferrogels play an essential role in the magnetoelastic behavior. ${ }^{20}$ Raikher and Stolbov ${ }^{21}$ discussed elongation of a magnetoelastic material under the action of a uniform external magnetic field.

A time-varying external magnetic field programmed to alter directions induces directional elasticity variations in a magnetic ferrogel implant and provides a means of control of mechanical properties. There is a concurrent magnetic effect too. When a magnetic field is applied, a ferrogel acquires a net magnetic moment due to the ordered orientation of particles in the field direction. This magnetic ordering is reversible. The material reverts to randomized orientation on switching off the external field. ${ }^{22}$ Several approaches can be adopted to take advantage of this property for contraceptive distribution and control. In one technique, a steady magnetic field leads to orientation and magnetic moment generation and a crossed time-varying field produces micro rotations of the magnetic entities and in consequence of the bulk polymeric material. These micro motions embed the contraceptive into the tissue fold in the lumen of the reproductive tract mucosa helping in long term retention of the compound.

Nanomicro-sized magnetic particles when in use for fertility control tend to agglomerate and adsorb to plasma proteins due to their large surface-to-volume ratio and magnetic properties. ${ }^{23}$ Therefore, surface coverage of magnetic particles $\mathrm{Fe}_{3} \mathrm{O}_{4}$ with a safe and effective polymer SMA significantly increases the stability and ensures proper distribution by eliminating agglomeration and adsorption to proteins. ${ }^{24-26}$ In line with Hoffman and colleagues' 27 observation that PVA coating of superparamagnetic iron oxide nanoparticles (PVA-SPION) reduced the cytotoxicity of native iron oxide to synovial cells of sheep melanoma cells, it is theoretically predictable that SMA coating of iron oxide will have no cytotoxic potential on of the iron oxide component.

Copper particles in the contraceptive introduce two functions. Copper, with its intrinsic high electrical conductivity, makes the overall compound electrically conductive in contrast to the purely polymeric RISUG which is a poor electrical conductor. Electromagnetic induction heating of the implant by means of an external microwave field is feasible. By heating, the contraceptive can be softened to facilitate removal for restoration of fertility. The second function of copper is to displace some specific molecules, for example, zinc, from the sperm membrane thereby lowering the fertilizing potential of the sperm. Copper lowers the fertility of ovum by displacement of proteins. ${ }^{28}$ Hence the presence of copper enhances the contraceptive action of Smart RISUG in both the male and female applications.

Extensive literature in the field reports a number of nanotech ferromagnetic compounds. These materials were synthesized for both biological and nonbiological uses. A review indicates that the properties do not match with the needs of fertility control; in particular a drug which can be implanted and controlled from outside the body by means of PMF. Although the spermicidal and tissue reaction properties of these materials have not been reported, a review of the basic chemical structure indicates that the compounds described in scientific literature would not have the desired combination of high spermicidal action together with low adverse tissue reaction. Efficacy as a spermicide has not been investigated. A close examination of the chemical structure suggests that the combination of desired properties will not be delivered by these products. Therefore the main objectives of our present research were to structure Smart RISUG and determine the feasibility of noninvasive control and to target this new drug into rat sperm cell membrane with the help of an impulse magnetizer. The physicochemical properties of this new compound were analyzed by differential scanning calorimetry (DSC); Fourier transform infrared spectroscopy (FTIR); high resolution X-ray diffractometery (HR-XRD); energy dispersive X-ray spectroscopy (EDS); and high resolution transmission electron microscopy (HRTEM). 


\section{Materials and methods \\ Materials}

Sourcing of the raw materials used in the study was: SMA from the RISUG Pilot Plant (Indian Institute of Technology, Kharagpur, India); $\mathrm{Fe}_{3} \mathrm{O}_{4}$ (Eisen (III) oxid, pulver $<5$ micron, 98\%) made by Sigma-Aldrich (GmbH, Germany). Cat.; 31, 006-9 make; copper powder - 625 mesh, APS 1-1.5 micron, 99\% (metals basis) obtained from Alfa Aesar (Shore Road, Heysham, Lancaster, UK); dimethyl sulphoxide (DMSO D2650, HYBRI-MAX ${ }^{\circledR}$, hygroscopic, FW 78.13) supplied by Sigma-Aldrich (Singapore); glutaraldehyde solution 2.5\% purchased from Merck CAS No. 111-30-8; phosphate buffer saline (PBS) procured from Merck (Darmstadt, Germany); ethanol, absolute $\left(\mathrm{CH}_{3}-\mathrm{CH}_{2}-\mathrm{OH}, \mathrm{AR}, \mathrm{MW}\right.$ 46.080) purchased from Hong Yang Chemical Corporation (China); osmic acid 2\% solution w/v for microscopy was obtained from ISO 9001-2000 certified CDH (P) Ltd. (New Delhi, India); araldite 506 epoxy resin, viscosity $\left(25^{\circ} \mathrm{C}\right)$ : $500-700 \mathrm{cps}$, weight per epoxide: $172-185$ was also purchased from Sigma-Aldrich (St. Louis, MO, USA); uranyl acetate and lead (II) citrate trihydrate for electron microscopy, Fluka grade, Mr1053.83 from Sigma Aldrich (UK); propidium iodide (PI) solution 1.0 MG/ML, P4864, FW 668.4 used for fluorescent staining purpose imported from Sigma-Aldrich (USA).

\section{Preparation of Smart RISUG compound}

SMA copolymer was synthesized as per the protocol given by the inventor mentioned in US patent 5,488,075. ${ }^{4}$ The new Cuproferrogel compound $\mathrm{Fe}_{3} \mathrm{O}_{4}-\mathrm{Cu}-\mathrm{SMA}-\mathrm{DMSO}$ or Smart RISUG was synthesized by adding $2 \%-15 \%(\mathrm{w} / \mathrm{v})$ iron oxide nanomicro-sized particles $(<5 \mu \mathrm{m}), 3 \%-8 \%(\mathrm{w} / \mathrm{v})$ copper nanomicro-sized particles and SMA together to the DMSO (1:8) under a dry XL grade nitrogen atmosphere. The nitrogen gas was first passed through a $0.2 \mu$ filter. Thereafter, the solution obtained was continuously stirred for 48 hours at $35^{\circ} \mathrm{C}$ adopting the method given by the inventor. ${ }^{18}$

\section{Animal maintenance}

With the approval of the Institutional Animal Ethics Committee, mature male albino rats of weight $>150 \mathrm{~g}$ were used for this experiment. Albino rats kept at the animal house of the Department of Biotechnology, Indian Institute of Technology, Kharagpur, India under condition as per recommendations of the Institutional Animal Subject Review Committee. Animals were maintained at approximately $25^{\circ} \mathrm{C}$ and $55 \%$ humidity with a $12 \mathrm{hr}$ light-12 hr dark cycle and were with standard chow and water ad libitium.

\section{Sperm cell collection}

Sperm cells were collected from the distal cauda epididymis of mature male albino rats, (weighing 150-200 g) after anesthetizing the animals with sodium barbiturate i.p. (30 mg/kg). About $1 \mathrm{~cm}$ stream of epididymal fluid was expelled from the cauda into $3 \mathrm{ml}$ of $0.1 \mathrm{M}$ PBS (pH 7.4) in a $35 \mathrm{~mm}$ polystyrene culture disk. ${ }^{29}$ The epididymal tissue was removed and the dish kept in incubator for 10 minutes. Sperm samples were then diluted to about $15-20 \times 10^{6}$ sperms $/ \mathrm{ml}$. The viable cells were collected by swim-up technique. ${ }^{30}$

\section{Sperm count, motility and viability}

Microscopic examination of the slides of sperm cells after interaction with RISUG and Smart RISUG at magnetic field of different pulse densities followed. A Neubauer cell counting chamber served as the calibrated grid for sperm counts. Sperm motility was assessed by a phase contrast microscope (Olympus CKX41 Optical Co., Shizuoka, Tokyo, Japan) equipped with a $40 \times$ objective and a Steves Digicams C-5060 wide zoom camera.

\section{Energy dispersive X-ray spectroscopy}

Smart RISUG is a chemically cross-linked Cuproferrogel, a colloidal dispersion of magnetic particles having superparamagnetic behavior and copper. Preferred particle form is a size less than $5 \mu$ diameter and being a mix of nano-sized and micro-sized $\mathrm{Fe}_{3} \mathrm{O}_{4}$ and $\mathrm{Cu}$ particles. EDS associated with X-ray microanalysis of the new compound was done with scanning microscope. (JEOL JSM-5800, OXFORD ISIS-300, NY, USA)

\section{Particle size analysis}

The particles size of iron oxide and the new drug Smart RISUG were observed with the particle size analyzer (MALVERN Instruments, Zetasizer nano ZS, Worcestershire, UK).

\section{Fourier transform infrared spectroscopy}

FTIR analysis of the RISUG and the new compound Smart RISUG was done with FTIR instruments, Thermo Nicolet NEXUS 870 Corporation, Brewster, New York, USA.

\section{High resolution transmission electron microscopy}

HRTEM studies were performed on a transmission microscope. (Model JEM 2100 Oxford Instruments, Oxfordshire, UK) High magnifications, bright field imaging, selected area electron diffraction (SAED) were performed on JEM 2100. 


\section{Atomic force microscopy}

Fixed sperm cells were immobilized on $0.01 \%$ poly-L-lysinecoated slides prior to analysis by noncontact mode atomic force microscopy (AFM; Thermo Microscopes, CP Research Model, and Sunnyvale, CA, USA). Control and Smart RISUG-treated samples were analyzed for changes by tapping mode AFM on the outer surface of sperms. A $50 \mu$ long silicon cantilever, with a force constant of $17.2 \mathrm{~N} / \mathrm{m}$ was vibrated near its resonance frequency of $320 \mathrm{kHz}$. The deviation of the vibration frequency from resonance depends on the tip-sample preparation and forms the data set providing the topography of the sperm cell.

\section{External pulsed magnetic field exposure system}

A laboratory designed and fabricated impulse magnetizer of up to $800 \mathrm{mT}$ field strength provided variable magnitude magnetic pulses for the investigations. This instrument basically consists of $2000 \mu \mathrm{F}$ capacitor bank charged to a voltage of $50 \mathrm{kV}$ by means of a high-turn ratio transformer and rectifier. The capacitor charge was switched on to a very low electrical resistance copper coil to generate the magnetic field. Field strength produced was monitored with a search coil connected to a $100 \mathrm{MHz}$ recording oscilloscope. Before commencing the experiment, freshly collected sperm sample was kept inside a test tube in front of the magnetic coil. On discharging the instrument, magnetic field of a specific pulse was produced depending on the rate of magnetization of the magnetic coil. The range of magnetic flux density produced by the instrument is $1 \mathrm{mT}$ (with main spectral band centered at $50 \mathrm{~Hz}$ to $800 \mathrm{mT}$ with centre frequency of $40 \mathrm{KHz}$. The magnetophoretic mobility of the compound was measured manually by observing the distance moved in time. Ingress of the Smart RISUG compound into sperm cell was observed with microscope and the specific pulse produced each time was calculated with oscilloscope as per the calibration of the magnetic search coil.

\section{Magnetophoretic mobility}

Magnetic field-mediated transport properties of the Smart RISUG compound are dependent on the magnetophoretic mobility which is a measure of cell magnetism that is magnetic force per bead $\left(\mathrm{F}_{\mathrm{b}}\right)$ and represented by

$$
F_{b}=\Delta \chi_{b} \cdot V_{b} \cdot S_{m}
$$

where $\chi_{b}=$ volumetric magnetic susceptibility of magnetic bead; $V_{b}=$ volume of magnetic particle; $S_{m}=$ quadrupole magnetic field strength/magnetic energy density. Magnetic force on each cell $\left(F_{m}\right)$ is

$$
F_{m}=A B C \cdot \beta \cdot F_{b}=\left(n_{1} \theta_{1} \lambda_{1)}\left(n_{2} \theta_{2} \lambda_{2}\right)\right.
$$

where $\beta=$ amplification factor (no of magnetic beads attached); $n_{1}=$ no of coating particles (here RISUG); $\lambda_{1}=$ fraction of coating particles (here RISUG); $\lambda_{1}=$ valency of binding particle (here RISUG); $n_{2}=$ no of magnetic particles (here Iron oxide); $\lambda_{2}=$ fraction of magnetic particles (here Iron oxide); $\lambda_{2}=$ valency of central particle (here Iron oxide). Therefore, drag force on each cell $\left(F_{d}\right)$ is

$$
F_{d}=\left(3 \pi \cdot D_{c} \cdot \eta\right) \cdot U_{c}
$$

where $D_{c}=$ diameter of cell; $\eta=$ viscosity of the medium; $U_{c}=$ induced velocity in the field.

\section{Statistical analysis}

Student's paired t-test was used to measure the difference between the samples. $\mathrm{P}<0.5$ was considered statistically significant. All values are given as mean \pm standard deviation (SD).

\section{Results}

\section{A. Smart RISUG co-polymer drug synthesis and characterization}

\section{Particle distribution}

Energy dispersive X-ray microanalysis (EDS) investigated arrangement of the $\mathrm{Fe}_{3} \mathrm{O}_{4}-\mathrm{Cu}-\mathrm{SMA}-\mathrm{DMSO}$ Cuproferrogel particles about $50 \mathrm{~nm}$ to $4 \mu$ (Figure 1). An approximately uniform size of SMA-DMSO-coated iron oxide particles and copper particles were observed. The drug particles appeared to be properly dispersed.

\section{Particle size analysis}

Figure 2A shows that average size of $\mathrm{Fe}_{3} \mathrm{O}_{4}$ particles used here is $201.2 \mathrm{~nm}$. Polydispersity index value (PI) of Smart RISUG (Figure 2B) after Cuproferrogel formation done with the particle size analyzer illustrates that the average size of Smart RISUG particles is $628.2 \mathrm{~nm}$.

\section{Transmittance peaks}

FTIR peaks of the $\mathrm{Fe}_{3} \mathrm{O}_{4}-\mathrm{Cu}-\mathrm{SMA}-\mathrm{DMSO}$ (Smart RISUG) compound found at $952 \mathrm{~cm}^{-1}, 1020 \mathrm{~cm}^{-1}, 1317 \mathrm{~cm}^{-1}$, $1650 \mathrm{~cm}^{-1}, 2917 \mathrm{~cm}^{-1}$, etc. indicated presence of RISUG molecule in the 'Smart RISUG' (Figure 3B). In addition to parent constituents SMA and DMSO, the significant peaks observed at $707 \mathrm{~cm}^{-1}, 1436 \mathrm{~cm}^{-1}$ indicated presence of nanomicro 


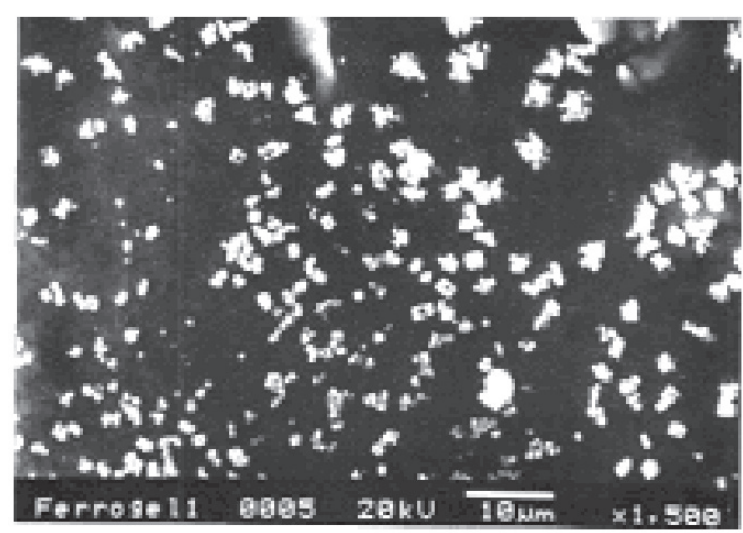

Figure I X-ray microanalysis of the $\mathrm{Fe}_{3} \mathrm{O}_{4}-\mathrm{Cu}$-SMA-DMSO (Smart RISUG) indicating arrangement of drug particles in the Cuproferrogel compound.

magnetic particles $\left(\mathrm{Fe}_{3} \mathrm{O}_{4}\right)$ and $3004 \mathrm{~cm}^{-1}$ peak indicated presence of copper particle in the new compound.

\section{External magnetic field-mediated control and interaction of Smart RISUG with sperm cell}

\section{Transport mechanism}

HRTEM showed that Smart RISUG particles which could enter sperm cell membrane were of $50 \mathrm{~nm}$ to $200 \mathrm{~nm}$ size, the range suitable for delivery in a reproductive tube as it neither enters the blood circulation nor gets away with the reproductive tube fluid. Figure 4A shows attachment of new compound particles to the sperm cell membrane at head region. Figures 4B and 4C track Smart RISUG particles inside the sperm cell. It is evident that particles either attach to cell membrane or enters only nearby cytoplasm without causing damage to the genetic material. This point is of considerable significance in terms of safe genotoxic potential of the drug in clinical application.

\section{Effect of external pulsed magnetic field exposure on Smart RISUG compound}

In physical magnetophoretic mobility experiments, the overall lengthening of the material kept in a test tube as a percentage of the pre PMF exposure length versus the magnetic field strength is shown. The relative displacement induced by nonuniform magnetic field can be $40 \%$ of the initial length (Figures 5A and 5B). The Smart RISUG drug mobility initiates above PMF of $80 \mathrm{mT}$. An abrupt increase in the lengthening in field strength of about $280 \mathrm{mT}$ provides a general guideline for the choice of optimal field strengths for biophysical experiments. Thus a significant magnetostriction took place and hysteresis was observed (Figure 5c).
One other experiment was conducted to determine optimum concentration of iron oxide. Taking different concentration ( $2 \%$ to $15 \%$ by weight) of iron oxide particles in the new compound wherein the threshold magnetic field for mobility for each concentration was estimated. It was observed that with increasing iron oxide concentration the threshold magnetic field required for movement initiation decreases. Suitable concentration of the iron oxide was found to be $8 \%$ to $12 \%$ by weight and threshold magnetic field strength required for its lengthening initiation was found to be $80 \mathrm{mT}$ (Figure 5D).

\section{Interaction of sperm cell with Smart RISUG} in the absence and presence of pulsed magnetic field produced by the impulse magnetizer

RISUG precipitate when made to attach to top of the test tube, it could not be transported into sperm cells kept at the bottom of tube either in presence or absence of PMF. Similarly, no significant change in the sperm count or sperm motility percentage occurred when Smart RISUG precipitate and diluted sperm cells are kept at the two ends of the test tube in the absence of PMF as in this case the Smart RISUG is not able to reach or enter the sperm cell membrane in order to facilitate any changes thereof.

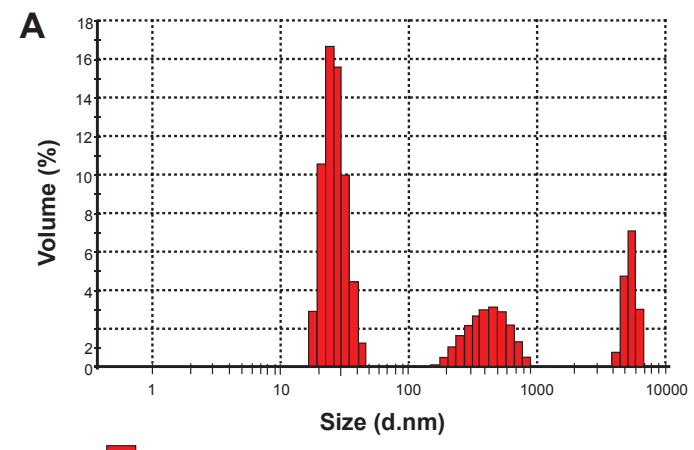

Mean with +/-1 Standard Deviation error bar

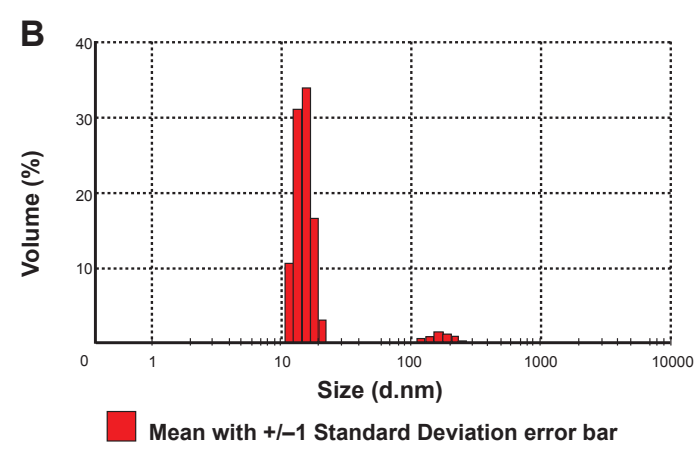

Figure 2 Particle size analysis. A) $\mathrm{Fe}_{3} \mathrm{O}_{4}$ particles are in three ranges; nanosize $(50 \mathrm{~nm}-100 \mathrm{~nm})$, microsize $(<I \mu \mathrm{m})$ and macrosize $(5-7 \mu \mathrm{m})$ B) Smart RISUG particles size Z- average is $628 \mathrm{~nm}$. 

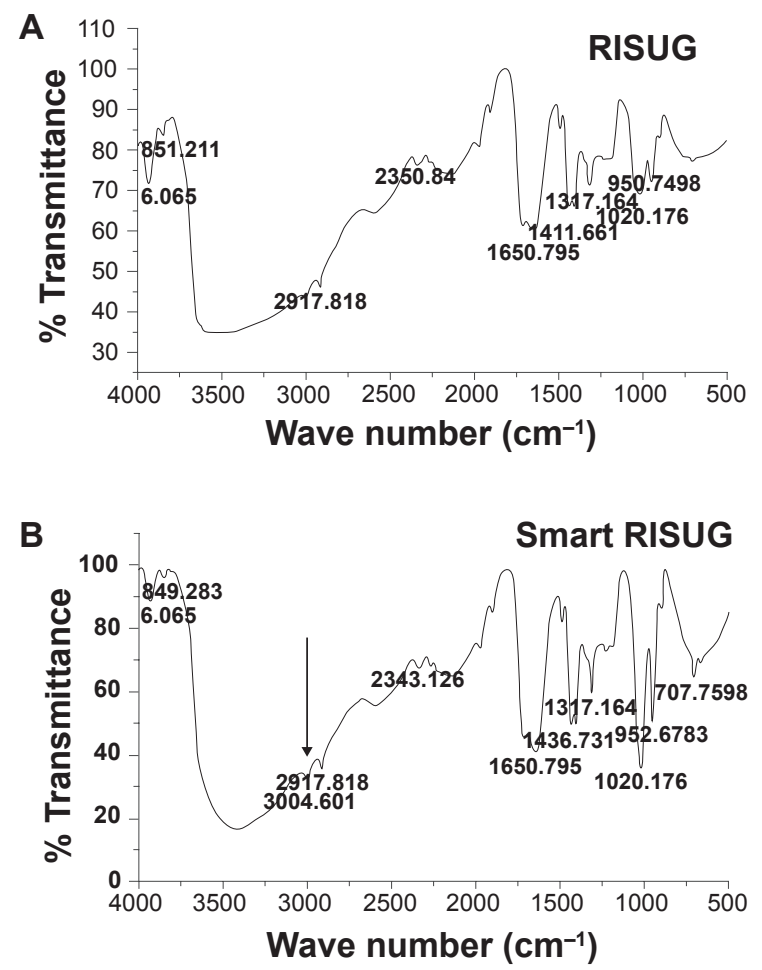

Figure 3 Fourier transform infrared spectroscopy. A) FTIR peaks for RISUG gel. B) FTIR peaks for Smart RISUG Cuproferrogel indicate presence of $\mathrm{Fe}_{3} \mathrm{O}_{4}$ and $\mathrm{Cu}$ particles embedded in matrix of parent compound RISUG (SMA-DMSO)

In the present study no significant variation in the sperm count and percentage of motile sperm were found when RISUG interacted with sperm cell either in presence or absence of PMF given for $30 \mathrm{~s}$ to $50 \mathrm{~s}$. The new contraceptive molecule started moving at $80 \mathrm{mT}$ but showed abrupt transformation at $280 \mathrm{mT}$ only. Table 1 reveals that Smart RISUG achieved abrupt transformation at about $280 \mathrm{mT}$ and it could be transported into sperm cell membrane at $760 \mathrm{mT}$ only. The interaction of rat sperm with Smart RISUG caused $30 \%$ decrease in sperm count and $70 \%$ decrease in sperm motility $(\mathrm{p}<0.05)$ (Table 1$)$.

\section{Drug-induced topological alteration}

AFM image of untreated rat sperm cells (Figure 6A) gave evidence of smooth surface topography throughout the length of the sperm cell membrane. A cluster of sperm cells with prominent head were observed with AFM. In contrast, sperm cells treated with Smart RISUG (Figure 6B) gave disintegrated surface topology with a complete loss of smoothness. The three-dimensional AFM image of the same showed curved tail and damage to the middle piece. A significant reduction in the cell height had been observed for treated sperm cells with AFM. An interesting observation here was the pattern of attachment of Smart
RISUG particles to sperm cell membrane complimenting HRTEM observation.

\section{Discussion}

The present study reveals magnetic field-directed $\mathrm{Fe}_{3} \mathrm{O}_{4}-$ $\mathrm{Cu}-\mathrm{SMA}-\mathrm{DMSO}$ Cuproferrogel drug interaction with sperm cells that may open new dimensions in the field of biomagnetic technology. RISUG action on sperm cells is not altered by PMF. PMF in the field strengths of $80-800 \mathrm{mT}$ applied for $30 \mathrm{~s}$ to $50 \mathrm{~s}$ does not have significant effect on the sperm. The change in the form of RISUG to that of Smart RISUG too does not alter the effect on sperm. That is, the concentrations of iron oxide and copper are sufficiently low as not to affect sperm function by their presence. Only the combination of the iron oxide-copper-PMF enhances the spermicidal action and hence the contraceptive efficacy of the parent RISUG compound. Moreover there is a threshold
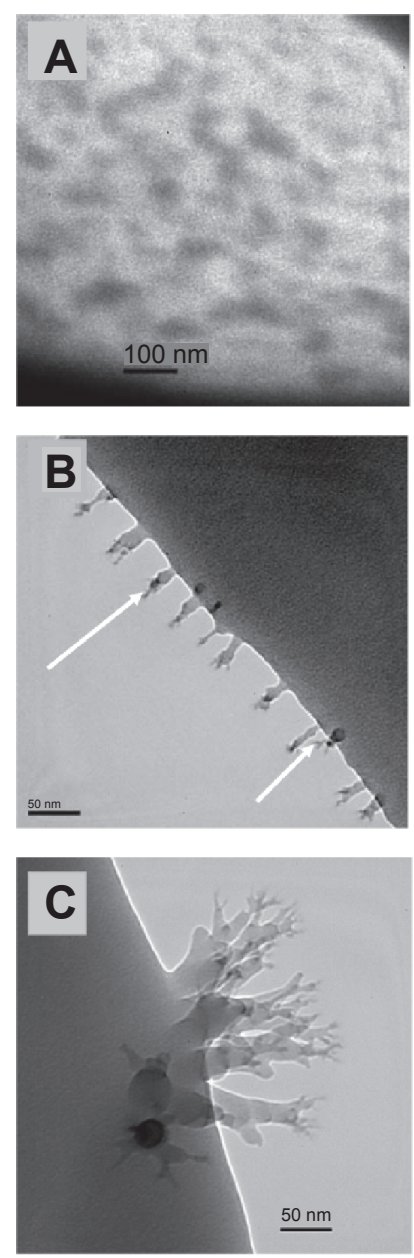

Figure 4 High resolution transmission electron microscopic (HRTEM) study of Smart RISUG compound's transport mechanism into sperm cell membrane. A) Surface of the rat sperm showing Smart RISUG particles attached to it. B, C) Mechanism of reaching of Smart RISUG particles into the sperm membrane. Arrows indicate passage of entry of drug into cell membrane. 

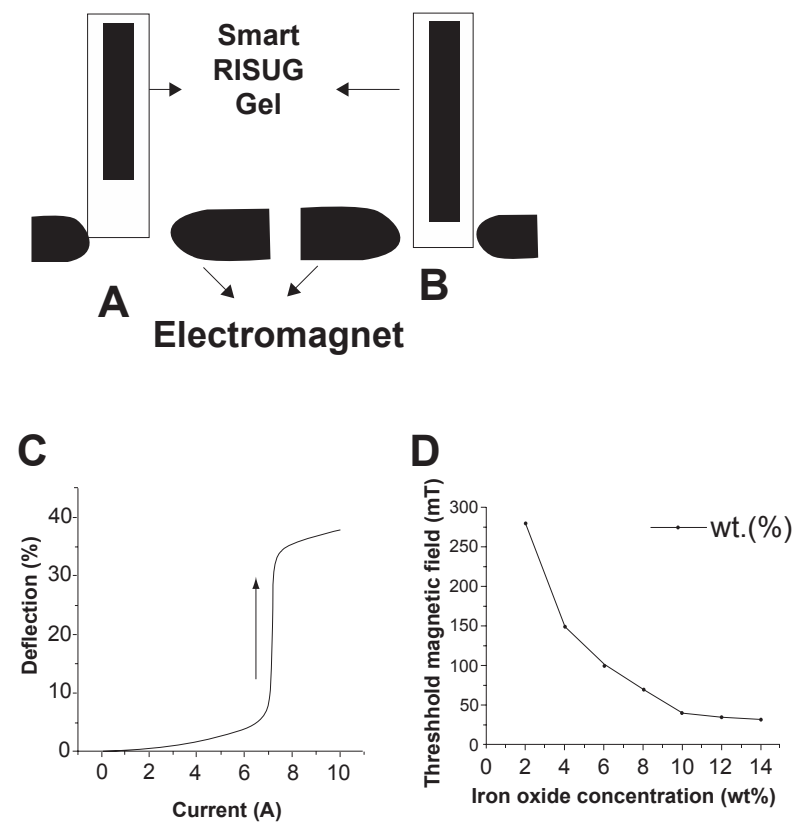

Figure 5 Response of Smart RISUG. A) No magnetic field. B) Electromagnet at the lower end caused Cuproferrogel elongation. C) Smart RISUG deflection $\Delta \mathrm{h} / \mathrm{h}_{0}(\%)$ versus current (A) given -a hysteresis graph obtained (D) Threshold magnetic field vs iron oxide concentration. The presence of $10 \%$ iron oxide causes threshold magnetic field effect in presence of external magnetic field.

iron oxide concentration above which the phenomena are manifest. Furthermore PMF of $760 \mathrm{mT}$ or above is required to induce the spermicidal enhancement potential. The about $30 \%$ decrease in the sperm count and about $70 \%$ decrease in the sperm motility percentage demonstrated in the present study imply that good contraceptive action may be realized with lower drug dose than the conventional RISUG.

HRTEM elucidates the entry mode of Smart RISUG particles into the sperm cell. Figures 4A and 4B illustrates that particles are not endocytosed by the sperm cells rather they attach to the sperm head surface or enters cytoplasm in vicinity of the sperm cell plasma membrane (Figure 4C) and thus inactivate the sperm cells. It is previously hypothesized that plasma membrane is directly involved in the mechanisms by which nonthermal extremely low frequency (ELF) electromagnetic fields exert their action on cell functions. ${ }^{31}$ Membrane disintegration of the sperm at the acrosomal region leads to leaching out of enzymes acrosin and hyaluronidase, thus rendering the sperm infertile, a mechanism similar to RISUG observed with AFM (Figure 6B).

Previous work on RISUG also reports that as sperm come into contact with the SMA-DMSO complex, the combination of positive and negative charges on the polymer surface causes the sperm membrane to burst. ${ }^{11}$ When co-polymer of styrene and maleic anhydride dissolved in DMSO was injected into the vas deferens of rats the morphological changes detected were confined to the mucosa. ${ }^{9}$ Just after injection, RISUG lowers the $\mathrm{pH}$ sufficiently to kill the sperm passing through. ${ }^{32,33}$ RISUG is presently undergoing extended phase III clinical trials in India ${ }^{2,34}$ after successfully completing phase I and phase II trials. ${ }^{33,35-37}$ The presence of common parent component SMA in Smart RISUG ensures long-term safety and safe reversibility of the drug.

For Smart RISUG drug synthesis the SMA copolymer is dissolved in solvent medium DMSO. Thereafter, magnetic material $\mathrm{Fe}_{3} \mathrm{O}_{4}$ is added to the polymer to impart magnetic properties and copper is added to obtain electrical conductivity and charge transfer capability that in turn affect structure of sperm/ovum by displacing specific molecules. Here, $\mathrm{Fe}_{3} \mathrm{O}_{4}$ added to SMA-DMSO facilitates noninvasive imaging as well as control of distribution of the drug in the reproductive tube under guidance of external magnetic field by enhancing contrast of drug with the body tissue. Use of magnetic particle iron oxide in Smart RISUG exploit two major advantages; their low toxicity to human beings and the possibility to use their outstanding properties by allowing the high magnetization of the iron oxide to target drugs to/from a specific cell through an external magnetic field. ${ }^{38,39}$ The addition of copper particles displaces specific molecules like zinc from sperm cell, reduces sperm motility and increases sperm abnormality. Both additives are uniformly dispersed in the base polymer and aggregation is prevented by suitable coating with crosslinked SMA and continuous stirring for 45-50 hrs.

The contraceptive preparation is taken in a $250 \mathrm{pl}$ syringe provided with a 23 gauge needle for injection into male/ female reproductive tube. In male a puncture is made in anterior scrotum to elevate vas deferens and then the needle is inserted into vas lumen. Smart RISUG has properties of electrical charge producing, $\mathrm{pH}$ lowering, molecular exchange property, charge transfer property and residual magnetism. The drug after injection swells and invaginates into the folds of the lumen to help retention but does not adhere to the tissue. When the pure polymeric RISUG is injected into the vas deferens the sperms present in the vas deferens at the time of injection are subjected to RISUG action and break-up. The fragments of the sperm are voided at the time of ejaculation. If on the other hand a compound such as Smart RISUG is injected into the vas deferens and concurrently PMF is applied the iron oxide enters the sperm membrane. A mosaic architecture of iron oxide and with it copper micro-strands corresponding to the sperm shape are formed (Guha, Pers Comm). These micro-strands enveloped 

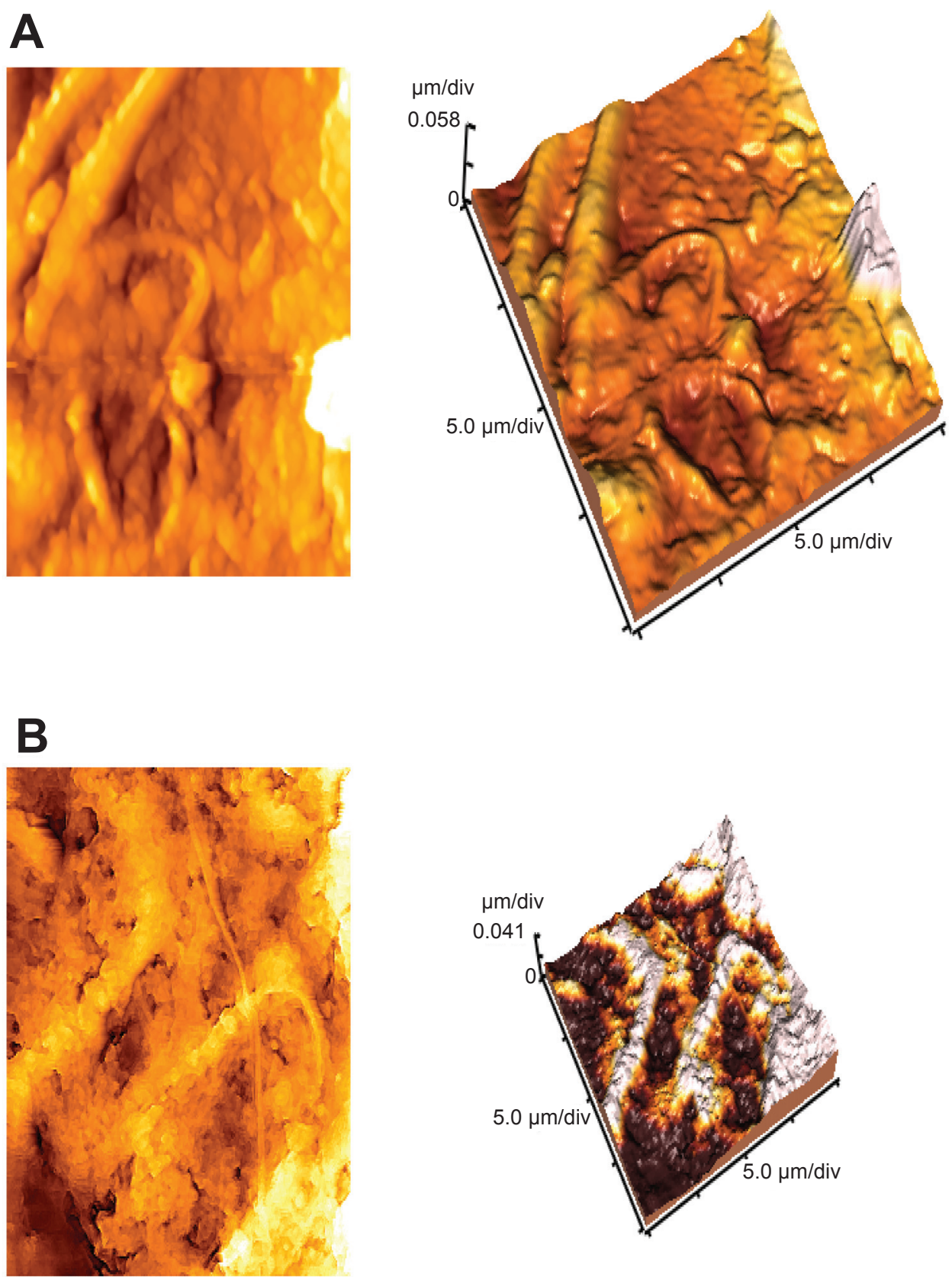

Figure 6 Atomic force microscopy (AFM) images of rat sperm. A) 2D and 3D images of untreated cells showing cluster cells with smooth surface. B) 2D and 3D images of $\mathrm{Fe}_{3} \mathrm{O}_{4}-\mathrm{Cu}-\mathrm{SMA}-\mathrm{DMSO}$ (Smart RISUG) treated cells showing blabs in plasma membrane, disintegration leading to enzyme leaching, shortened height, and curved tail.

by sperm proteins and lipids persist and create a large surface of electrical charge interaction for the destruction of sperms passing later on even without PMF. In this manner the spermicidal action is enhanced in a sustained manner. The removal as proposed in present paper is easily possible by using magnetic properties of the contraceptive to propel the drug for fertility restoration by external magnetic field.

This study explains that the new contraceptive molecule $\mathrm{Fe}_{3} \mathrm{O}_{4}-\mathrm{Cu}-\mathrm{SMA}-\mathrm{DMSO}$ (Smart RISUG) starts moving as a system in the presence of external PMF. The magnetic field exposure system developed for this study produced flux density up to $800 \mathrm{mT}$. As each particle is the elementary carrier of a magnetic moment, similar is the case for Smart RISUG particles that couple the shape of the gel to the PMF. On application of varying PMF, as the current of the electromagnet increases there is small movement of the new compound followed by an abrupt rise in the transport properties (Figure 5C). At the threshold magnetic field $80 \mathrm{mT}$ given for periods of $50 \mathrm{~s}$, the Smart RISUG compound starts movement and at about $760 \mathrm{mT}, 50 \mathrm{~s}$, the compound 
Table I Effect of external pulsed magnetic field (PMF)-mediated Smart RISUG exposure on the sperm cell count and motility where respective drugs and sperm cells are kept at the two different ends of reproductive tube model for this noninvasive control experiment

\begin{tabular}{|c|c|c|}
\hline & $\begin{array}{l}\text { Sperm cell } \\
\text { count }\left(10^{6} / \mathrm{ml}\right)\end{array}$ & $\begin{array}{l}\text { Sperm cell } \\
\text { motility (\%) }\end{array}$ \\
\hline I. C (absence of PMF) & $25.05 \pm 1.10$ & $68.37 \pm 3.97$ \\
\hline 2. C (presence of PMF) & $24.89 \pm 6.54$ & $68.13 \pm 2.89$ \\
\hline 3. RISUG (absence of PMF) & $24.87 \pm 1.13$ & $67.90 \pm 6.32$ \\
\hline 4. RISUG (presence of PMF) & $24.32 \pm 5.25$ & $67.80 \pm 4.66$ \\
\hline 5. Smart RISUG (absence of PMF) & $24.87 \pm 1.32$ & $65.29 \pm 2.24$ \\
\hline 6. Smart RISUG treated (presence of PMF) & $17.74 \pm 0.98$ & $45.12 \pm 4.13$ \\
\hline
\end{tabular}

Note: Data presents the mean \pm SEM of five samples $(p<0.05)$.

Abbreviations: $C$, control; PMF, pulsed magnetic field.

could enter the sperm cell membrane (Figure 4A). Transport occurs instantaneously and disappears abruptly when the external magnetic field is removed.35\%-45\% lengthening of the Smart RISUG drug with respect to pre-PMF length could be produced by applying a nonuniform magnetic field (Figure 5B).

EDS-X ray spectroscopy analysis observed the proper dispersion of Smart RISUG Cuproferrogel particles (Figure 1). FTIR analysis transmittance peaks of the $\mathrm{Fe}_{3} \mathrm{O}_{4}-\mathrm{Cu}-\mathrm{SMA}-\mathrm{DMSO}$ (Smart RISUG) found at $952 \mathrm{~cm}^{-1}$, $1020 \mathrm{~cm}^{-1}, 1317 \mathrm{~cm}^{-1}, 1650 \mathrm{~cm}^{-1}$, and $2917 \mathrm{~cm}^{-1}$ indicates the presence of SMA-DMSO molecule in the 'Smart RISUG'. In addition to these, the peaks at $707 \mathrm{~cm}^{-1}, 1436 \mathrm{~cm}^{-1}$, and $3004 \mathrm{~cm}^{-1}$ indicate that the nanomicro-sized $\mathrm{Fe}_{3} \mathrm{O}_{4}$ and $\mathrm{Cu}$ particles has been encapsulated by the SMA-DMSO molecule (Figures 3A, 3B).

Our experimental and theoretical studies indicated that in the absence of magnetic field neither RISUG nor Smart RISUG is able to interact with the sperm cells. Significant transport properties of Smart RISUG compound can only be observed when the applied magnetic field is above the threshold value. Present fertility control technology of magnetic field-mediated sperm interaction of the new contraceptive molecule Smart RISUG needs further study for any possible toxicity chances before applying this technology to human subjects even though the moderate amount of $\mathrm{Fe}_{3} \mathrm{O}_{4}$ being used here does not affect sperm functionality. ${ }^{27,40}$ However, initial studies of Smart RISUG on both gonad weight analysis and sperm anomaly assay proves in vivo safety and efficacy of the new drug in rat. ${ }^{17}$ Present study suggest use of new drug Smart RISUG in order to facilitate better spermicidal action, control of distribution of the fertility control agent implanted inside the male or female reproductive tube and noninvasive detection by application of external magnetic field.

\section{Acknowledgments}

This work was supported by the Ministry of Health and Family Welfare, Government of India (M11011/2/2008-FP). The authors report no conflicts of interest in this work. We acknowledge Central Research Facility, Indian Institute of Technology, Kharagpur and Mr SN Jha for their experimental contribution.

\section{References}

1. Trussell J. Contraceptive efficacy. In: Hatcher RA, Trussell J, Nelson AL, Cates W, Stewart FH, Kowal D, editors. Contraceptive technology. 19th revised edition. New York, NY: Ardent Media; 2007; p. 747-826.

2. Sharma RS, Rajanna A, Singh BK, Mathur AK, Mukherjee AK. Current status of development of RISUG: an intravasal injectable male contraceptive. In: Sharma RS, Rajanna A, Rajalakshmi M, editors. Proceedings of the Conference on Recent Advances and Challenges in Reproductive Health Research. New Delhi, India: Indian Society for the Study of Reproduction and Fertility (ISSRF); 2007 Feb. 19-21. 2007; p. 127-140.

3. Sharma S, Sen P, Guha SK, Mukhopadhyay SN. Microbicidal male contraceptive-RISUG induced morphostructural damage in $E$ coli Colloids Surf B Biointerfaces. 2003;32:43-50.

4. Guha SK. Contraceptive for use by male. United States Patent: 1996 $5,488,075$.

5. Manivannan B, Mishra PK, Lohiya NK. Ultrastructural changes in the vas deferens of langur monkeys Presbytis entellus entellus after vas occlusion with styrene maleic anhydride and after its reversal. Contraception. 1999;59:137-144.

6. Manivannan B, Bhande SS, Panneerdoss S, Sriram S, Lohiya NK. Safety evaluation of long-term vas occlusion with styrene maleic anhydride and its non-invasive reversal on accessory reproductive organs in langurs. Asian J Androl. 2005;7:195.

7. Sethi N, Srivastava RK, Singh RK. Safety evaluation of a male injectable antifertility agent styrene maleic anhydride, in rats. Contraception. 1989;39:217-226.

8. Sethi N, Srivastava RK, Nath D, Singh RK. Preclinical toxicity study of a male injectable antifertility agent (styrene maleic anhydride) in rhesus monkeys, Macaca mulatta. J Med Primatol. 1991;20:89-93.

9. Verma K, Misro MM, Singh H, Mahajan S, Ray AR, Guha SK. Histology of the rat vas deferens after injection of a non-occlusive chemical contraceptive. J Reprod Fertility. 1981;63:539-542.

10. Chaudhury K, Sharma U, Jagannathan NR, Guha SK. Effect of a new injectable male contraceptive on the seminal plasma amino acids studied by proton NMR spectroscopy. Contraception. 2002;66:199-204. 
11. Chaudhury K, Bhattacharya AK, Guha SK. Studies on the membrane integrity of human sperm treated with a new injectable male contraceptive. Hum Reprod. 2004;19:1826-1830.

12. Sharma U, Chaudhury K, Jagannathan NR, Guha SK. A proton NMR study of the effect of a new intravasal injectable male contraceptive RISUG on seminal plasma metabolites. Reproduction. 2001;122:431-436.

13. Sethi N, Srivastava RK, Nath D, Singh RK. Teratological evaluation of an injectable male antifertility agent, styrene maleic anhydride, in rat. Int J Fertil. 1992;37:183-187.

14. Koul V, Srivastava A, Guha SK. Reversibility with sodium bicarbonate of styrene maleic anhydride, an intravasal injectable contraceptive, in male rats. Contraception. 1998;58:227-231.

15. Lohiya NK, Manivannan B, Mishra PK. Repeated vas occlusion and non-invasive reversal with styrene maleic anhydride for male contraception in langur monkeys. Int J Androl. 2000;23:36-42.

16. Lohiya NK, Manivannan B, Mishra PK, Sriram S, Bhande SS, Panneerdoss S. Preclinical evaluation for noninvasive reversal following long-term vas occlusion with styrene maleic anhydride in langur monkeys. Contraception. 2005;71:214-226.

17. Jha RK, Jha PK, Rana SVS, Guha SK. Spermicidal action of styrene maleic anhydride polyelectrolyte in combination with magnetic and electrically conductive particles. Int J Pharmacol. 2009;5(1):1-12.

18. Guha SK. An improved reversible contraceptive for male and female. Canada Patent: 2007. 2367414.

19. Gaganidze E, Esquinazi $P$, Ziese M. Vibrating ferromagnets in a magnetic field. Journal of Alloys and Compounds. 2000;310:144-152.

20. Zrínyi M, Barsi L, Büki A. Ferrogel: a new magneto-controlled elastic medium. Polymer Gels and Networks. 1997;5:415-427.

21. Raikher YL, Stolbov OV. Magnetodeformational effect in ferrogel samples. JMMM. 2003;258-259, 477-479.

22. Lebedev VT, Torok G, Cser L, et al. Magnetic phase ordering in ferrogels under applied field. JMMM. 1999;201:136-139.

23. Zhang Y, Kohler N, Zhang M. Surface modification of superparamagnetic magnetite nanoparticles and their intracellular uptake. Biomaterials. 2002;23:1553-1561.

24. Gruttner C, Rudershausen S, Teller J. Improved properties of magnetic particles by combination of different polymer materials as particle matrix. J Magn Magn Mater. 2001;225:1-7.

25. Gupta AK, Curtis A. Surface modification of superparamagnetic iron oxide nanoparticles and their intracellular uptake. Eur Cells Mater. 2002;4:101-102.

26. Schopf B, Neuberger T, Schulze K, et al. Methodology description for detection of cellular uptake of PVA coated superparamagnetic iron oxide nanoparticles (SPION) in synovial cells of sheep. JMMM. 2005;293:419.
27. Hofmann H, Juillerat J, Petri-Fink A, Chastellain M. Development of biocompatible functionalized superparamagnetic iron oxide nanoparticles for human cancer cell uptake. Eur Cell Mater. 2004;7:14.

28. Roblero L, Guadarrama A, Lopez T, Zegers-Hochschild F. Effect of copper ion on the motility, viability, acrosome reaction and fertilizing capacity of human spermatozoa in vitro. Reprod Fertil Dev. 1996;8:871-874.

29. Gravance CG, Garnen DL, Miller MG, Berger T. Fluorescent probes and flow cytometry to assess rat sperm integrity and mitochondrial function. Reprod Toxicol. 2001;15:5-10.

30. Younglai EV, Holt D, Brown P, Jurisicova A, Casper RF. Sperm swim-up techniques and DNA fragmentation. Hum Reprod. 2001;16:1950-1953.

31. Marinelli F, Bersani F, Ognibene A, Matteuci A, Santi S, Maraldi NM. Effect of the extremely low frequency (ELF) magnetic fields on cell membrane. Eng Med Biol Soc. 1992;1:309-311.

32. Guha SK, Ansari S, Anand S, Farooq A, Misro MM, Sharma DN. Contraception in male monkeys by intra-vas deferens injection of a pH lowering polymer. Contraception. 1985;32:109-118.

33. Misro M, Guha SK, Singh H, Mahajan S, Ray AR, Vasudevan P. Injectable non-occlusive chemical contraception in the male-I. Contraception. 1979;20:467-473.

34. Guha SK. Biophysical mechanism-mediated time-dependant effect on sperm of human and monkey vas implanted polyelectrolyte contraceptive. Asian J Androl. 2007;9:221-227.

35. Chaki SP, Das HC, Misro MM. A short-term evaluation of semen and accessory sex gland function in phase III trial subjects receiving intravasal contraceptive RISUG. Contraception. 2003;67:73-78.

36. Guha SK, Singh G, Anand S, Ansari S, Kumar S, Koul V. Phase I clinical trial of an injectable contraceptive for the male. Contraception. 1993;48:367-375.

37. Guha SK, Singh G, Ansari S, et al. Phase II clinical trial of a vas deferens injectable contraceptive for the male. Contraception. 1997;56:245-250.

38. Marchal G, Van Hecke P, Demaerel P, Decrop E, Kennis C, Schueren E. Detection of liver metastases with superparamagnetic iron oxide in 15 patients: results of MR imaging at 1.5T. Am J Roentgen. 1989; 152:771-775.

39. Lubbe AS, Bergemann C, Riess H, Schriever F, Reichardt P, Possinger K. Preclinical experiences with magnetic drug targeting: tolerance and efficacy. Cancer Res. 1996;56:4694-4701.

40. Makhluf SBD, Qasem R, Rubinstein S, Gedanken A, Breitbart H. Loading magnetic nanoparticles into sperm cells does not affect their functionality. Langmuir. 2006;22:9480-9482. 